\title{
CONCENTRACIÓN Y ACUMULACIÓN DE NUTRIMENTOS EN LA BIOMASA AÉREA DE PLANTACIONES DE TECA DE 3 A 18 AÑOS EN LA CUENCA DEL CANAL DE PANAMÁ
}

\author{
Rafael Murillo ${ }^{1 / *}$, Alfredo Alvarado $^{* *}$, Jean Mark Verjans ${ }^{* * *}$ \\ Palabras clave: Tectona grandis; concentración foliar; macronutrimentos; micronutrimentos; acumulación. \\ Keywords: Tectona grandis; macronutrients; micronutrients; foliar concentration; accumulation.
}

Recibido: 05/08/14

\section{RESUMEN}

Se colectaron muestras de los compartimentos de la biomasa aérea (tronco, corteza, ramas primarias, ramas secundarias y hojas) de 16 árboles dominantes de plantaciones de teca cuyo rendimiento en volumen varió de 9,4 a $13,3 \mathrm{~m}^{3} \mathrm{ha}^{-1} \cdot$ año $^{-1}$, entre los 3 a 18 años de edad, respectivamente, en la cuenca del Canal de Panamá, con el propósito de determinar la concentración y acumulación de macronutrimentos $(\mathrm{N}, \mathrm{K}, \mathrm{Ca}, \mathrm{Mg}, \mathrm{P}$ y S) y micronutrimentos (Fe, $\mathrm{Mn}, \mathrm{Zn}, \mathrm{Cu}$ y B) por compartimento y por árbol, en suelos mayormente rojos, arcillosos y ácidos, clasificados como Ultisoles. Se ajustaron funciones de regresión para estimar la acumulación de cada nutrimento según la edad. La biomasa seca promedio del tronco de 3 a 18 años representó el 59,6\% (C.V. 5\%) de la biomasa seca total, mientras que las ramas primarias el $16,6 \%$, la corteza el 9,4\%, las hojas el 7,9\% y las ramas secundarias el 6,5\%. Los macronutrimentos de mayor concentración según el compartimento fueron el $\mathrm{Ca}$ $(2,01 \%)$ en la corteza, y el N $(1,98 \%)$ en las hojas. Los micronutrimentos de mayor concentración fueron el Fe (767 mg. $\left.\mathrm{kg}^{-1}\right)$, Mn (60 mg.kg $\left.{ }^{-1}\right)$ y Zn (50 mg. $\mathrm{kg}^{-1}$ ), todos en la corteza. La acumulación de macronutrimentos a la edad de 18 años fue de

\footnotetext{
1 Autor para correspondencia. Correo electrónico: rafael.murillo.cruz@una.cr

* Universidad Nacional de Costa Rica, Heredia, Costa Rica.
}

Aceptado: $10 / 03 / 15$

\begin{abstract}
Concentration and accumulation of nutrients in the aerial biomass of teak plantations 3 to 18 old, in the Panama Canal watershed. Tissue samples from aerial biomass compartments (bark, wood, primary and secondary branches, and foliage) were taken from 16 dominant trees of teak in plantations of the Panama Canal watershed, whose volume yield ranged between 9.4 and $13.3 \mathrm{~m}^{3} \mathrm{ha}^{-1}$. year $^{-1}$ at ages 3 and 18 years, respectively, growing in clayey, red, and acid Ultisols. Wet and dry weight of the different tissues was measured and subsamples taken to be analyzed for macronutrients $(\mathrm{N}, \mathrm{K}, \mathrm{Ca}, \mathrm{Mg}, \mathrm{P}$ and $\mathrm{S}$ ) and micronutrients ( $\mathrm{Fe}, \mathrm{Mn}, \mathrm{Zn}, \mathrm{Cu}$ and $\mathrm{B}$ ). Regression analyses allowed to relate nutrients accumulation with tree age. Dry biomass of the wood was 59.6\% (C.V. 5\%) of total dry biomass, while primary branches, bark, foliage, and secondary branches represented 16.6, 9.4, 7.9, and 6.5, respectively. Larger concentrations of macronutrients were $\mathrm{Ca}(2.01 \%)$ found in the bark, and $\mathrm{N}$ in the foliage (1.98\%). As for micronutrients, larger concentrations were found in the bark, in the order of $\mathrm{Fe}\left(767 \mathrm{mg} \cdot \mathrm{kg}^{-1}\right), \mathrm{Mn}$ $\left(60 \mathrm{mg} \cdot \mathrm{kg}^{-1}\right)$ and $\mathrm{Zn}\left(50 \mathrm{mg} \cdot \mathrm{kg}^{-1}\right)$. At 18 years of
\end{abstract}

\footnotetext{
* Universidad de Costa Rica, San José, Costa Rica.

*** Ecoforest Panamá.
} 
15,9 kg.árbol ${ }^{-1}(7,3 \mathrm{~kg}$ de Ca; $3,9 \mathrm{~kg}$ de N; $2,6 \mathrm{~kg}$ de $\mathrm{K} ; 1,0 \mathrm{~kg}$ de $\mathrm{Mg} ; 0,7 \mathrm{~kg}$ de $\mathrm{P}$ y $0,4 \mathrm{~kg}$ de $\mathrm{S}$ ) y la de micronutrimentos fue de $124 \mathrm{~g}$ ( $89 \mathrm{~g}$ de Fe; 18 g de $\mathrm{Zn} ; 9$ g de B; 5 g de Mn y 3 g de $\mathrm{Cu}$ ).

\section{INTRODUCCIÓN}

\section{Biomasa Aérea}

La biomasa aérea de la teca (el tronco desde el nivel del suelo, la corteza, las ramas primarias y secundarias y las hojas) se ha estudiado principalmente en África y Asia (Kaul et ál. 1979, Nwoboshi 1984, Negi 1990, Zech y Kaupenjohann 1990, Negi et ál. 1995, Drechsel y Zech 1991, Koppad y Rao 2003, Mbaekwe y Mackenzie 2008, Kumar et ál. 2009, Kumar 2009) y recientemente en América (Barroso et ál. 2005, Vieira 2006, Guimaraes et ál. 2010, Portuguez 2012). Portuguez (2012), encontró que la biomasa seca por árbol de teca en zonas de clima estacionalmente seco en Costa Rica varió desde $4 \mathrm{~kg}$ (1,3 años) hasta $700 \mathrm{~kg}$ (17,1 años). La distribución de la materia seca total entre las diferentes partes aéreas del árbol, cambia con la edad, de tal forma que la proporción del tronco y las ramas tienden a aumentar mientras que la proporción de hojas tiende a disminuir (Nwoboshi 1983, Mbaekwe y Mackenzie 2008, Kumar 2009, Portuguez 2012). La biomasa por árbol joven de teca puede disminuir por la competencia de malezas por espacio, agua y nutrimentos así como por la carencia de algunos nutrimentos como el Ca y $\mathrm{N}$ en el suelo (Barroso et ál. 2005) y aumentar o disminuir por la aplicación de fertilizantes (Guimarães et ál. 2010) y la realización de podas y raleos adecuados. Vieira (2006) encontró que la biomasa seca aérea por unidad de superficie de la teca disminuye con el aumento del espaciamiento entre árboles, sin embargo, la biomasa seca aérea por árbol aumenta con el incremento en age accumulation of macronutrients was $15.9 \mathrm{~kg}$. tree $^{-1}(7.3 \mathrm{~kg} \mathrm{Ca}, 3.9 \mathrm{~kg} \mathrm{~N}, 2.6 \mathrm{~kg} \mathrm{~K}, 1.0 \mathrm{~kg} \mathrm{Mg}$, $0.7 \mathrm{~kg} \mathrm{P}$ and $0.4 \mathrm{~kg} \mathrm{~S}$ ) and $124 \mathrm{~g}$ of micronutrients (89 g Fe, $18 \mathrm{~g} \mathrm{Zn}, 9 \mathrm{~g} \mathrm{~B}, 5 \mathrm{~g} \mathrm{Mn}$ and $3 \mathrm{~g} \mathrm{Cu}$ ).

espaciamiento hasta un máximo de espaciamiento de $4 \times 3$ m cuando tiende a disminuir. Según varios autores la tasa de biomasa aérea de la teca (biomasa seca árbol $\cdot$ edad $^{-1}$ ) varía según la edad y la calidad del sitio entre 1,7 (Kumar 2009), 2,7 (Nwoboshi 1983), 8,0 (Kurmar et ál. 2009) y 39,5 $\mathrm{kg}$ árbol $^{-1} \cdot \mathrm{año}^{-1}$ (Nwoboshi 1984) en árboles de 1; 7,5; 10 y 13 años, respectivamente.

La distribución de la biomasa aérea seca acumulada por árbol de teca es variable y puede depender de la calidad de sitio forestal, ya que en sitios de mayor productividad en Nigeria (Nwoboshi 1983) un $88 \%$ de la biomasa se localizó en el tronco y la corteza, el restante $12 \%$ en ramas y follaje, mientras que en sitios de baja productividad en la India (Kaul et ál. 1979) reportó una distribución de un $57 \%$ en el tronco y corteza y un $43 \%$ en las ramas y el follaje.

Es importante considerar la distribución de la biomasa aérea en árboles de plantación debido a las repercusiones que podrían tener los raleos y la cosecha final en el balance de nutrimentos del sistema. Egnell y Ahnlund (2015) encontraron que la extracción del $100 \%$ de la biomasa en un raleo temprano en rodales de Pino Scots, causó una disminución de un $4 \%$ en el crecimiento de los árboles remanentes durante los siguientes 10 años. La tendencia mundial actual se basa en el aumento de la productividad forestal, la cual puede incrementar la cosecha anual hasta en un 19\% (Chandra et ál. 2011), lo que significa en una extracción mayor de biomasa y de nutrimentos $y$ posiblemente en un menor tiempo debido al aumento de la demanda de sustitutos de los combustibles fósiles. 
Concentración de Nutrimentos en la Biomasa Aérea

La concentración de nutrimentos en los compartimentos aéreos (tronco, corteza, ramas, hojas) no presenta gran variación respecto de la edad de los árboles, pero si es diferente entre los compartimentos (Nwoboshi 1984, Negi 1990, Negi et ál. 1995) y también puede ser afectada por la fenología (Portuguez 2012). La mayor concentración de nutrimentos en los compartimentos de la teca ocurre en las partes reproductivas, luego el follaje, seguido de las ramas, la corteza y en menor concentración en el tronco (Kumar et ál. 2009, Portuguez 2012).

El orden de concentración de macronutrimentos reportado, es variable, ya que en plantaciones de 3,5 años en Panamá (Mollinedo 2003, Mollinedo et ál. 2005) sigue el orden $\mathrm{K}>\mathrm{Ca}>\mathrm{Mg}>\mathrm{P}(1,33 ; 0,99 ; 0,34$ y $0,15 \%$ respectivamente), mientras que el orden de la concentración de macronutrimentos en la mayoría de los compartimentos aéreos de la teca en Guanacaste, Costa Rica es $\mathrm{Ca}>\mathrm{K}>\mathrm{N}>\mathrm{Mg}>\mathrm{P}>\mathrm{S}$ (Portuguez 2012).

La concentración de nutrimentos podría estar influida por la calidad del sitio, ya que en suelos rojos y ácidos de Benín se ha encontrado deficiencias de $\mathrm{K}$ foliar en las plantaciones de teca (Zech y Kaupenjohann 1990) mientras que en Ticoporo, Venezuela se han encontrado concentraciones de $\mathrm{K}$ foliar $(1,15 \%)$ en suelos moderadamente drenados que son superiores a las concentraciones halladas en sitios de drenaje pobre $(0,21 \%)$ (Hernández et ál. 1993).El crecimiento de la teca es mejor en suelos con concentración de Ca mayor a $15 \mathrm{cmol}^{-\mathrm{l}^{-1}}$, saturación de Ca mayor a $69 \%$, concentración de $\mathrm{K}$ mayor a 0,18 cmol. $^{-1}$ y concentración de P mayor de 6 mg..$^{-1}$ (Thiele 2008).

En el Oeste de África la concentración foliar de $\mathrm{N}$ y $\mathrm{P}$ en árboles de teca de 4 a 6 años decrece con la edad, mientras que la concentración de otros elementos como $\mathrm{K}, \mathrm{Mg}, \mathrm{Zn}$ y $\mathrm{Cu}$ permanece relativamente estable (Drechsel y Zech 1994). Las curvas de concentración foliar de N, P y K en relación con la edad de los árboles elaboradas en Costa Rica manifiestan una tendencia a disminuir hasta una edad de 8 años, mientras que las concentraciones foliares de $\mathrm{Ca}$, $\mathrm{Mg}, \mathrm{Mn}$ y $\mathrm{Zn}$ tienden a aumentar con la edad (Montero 1999).

La información disponible sobre concentración de micronutrimentos en los compartimentos de la biomasa aérea de árboles de teca es mucho menor que la de macronutrimentos. La evaluación de micronutrimentos foliares hecha en India (Negi et ál. 1995) para teca de 1 a 20 años, muestra valores superiores de $\mathrm{Fe}$ y $\mathrm{Zn}$ y semejantes para el Mn, en comparación con los datos de Panamá en árboles de 3,5 años (Mollinedo et ál. 2005); sin embargo, los valores guardan el mismo orden de concentración. Osundina y Osonubi (1989) encontraron que las concentraciones foliares de $\mathrm{Fe}$ y $\mathrm{Mn}$ en árboles de teca de menos de un año podrían aumentar hasta $400 \%$ y $186 \%$ respectivamente bajo condiciones de anegamiento y eventualmente alcanzar niveles tóxicos para la planta.

Portuguez (2012) menciona que los valores promedio de los microelementos en los diferentes compartimentos de la biomasa aérea de plantaciones de teca en Guanacaste, Costa Rica sigue el orden descendente de concentración $\mathrm{Fe}>\mathrm{Zn}>\mathrm{Mn}>\mathrm{B}>\mathrm{Cu}$ con valores marginales solamente de Mn y que las mayores concentraciones de elementos se encontraron en la corteza y el follaje, de manera intermedia en las ramas secundarias y primarias y en mucho menor cantidad en la madera. En el mismo estudio se encontró que la concentración de Fe en los diferentes compartimentos de la biomasa aérea y atribuible a contaminación atmosférica o a que su detección a nivel de laboratorio, al igual que la de los demás elementos menores considerados en el presente trabajo, presenta un porcentaje de variación entre 10 y 15\% (Bertsch et ál. 2005). La concentración $\mathrm{Zn}$ en todos los compartimentos de la biomasa aérea es baja y dependiente del contenido disponible del elemento y la profundidad efectiva del suelo (Portuguez 2012), a pesar de que la teca tiene capacidad de absorber $\mathrm{Zn}$ en cantidades elevadas (Prasanna et ál. 2006). Portuguez (2012) 
menciona que la concentración de Mn tiende a decrecer hasta los 4 años cuando se estabiliza en el follaje y en las ramas primarias y se encontró a nivel marginal por medio de los criterios desarrollados por Dreschel y Zech (1991). La concentración de $\mathrm{B}$ en todos los compartimentos de la biomasa aérea en árboles de teca, excepto en la corteza presenta poca variación con la edad y se encontró que varía entre $15-45 \mathrm{mg} \cdot \mathrm{kg}^{-1}$ en el follaje y el tronco (Portuguez 2012). La concentración de $\mathrm{Cu}$ en los diferentes compartimentos de la biomasa aérea de la teca son ligeramente más elevadas en el follaje y las ramas secundarias y tienden a disminuir lentamente con la edad de los árboles.

\section{Acumulación de Nutrimentos en la Biomasa Aérea}

La acumulación de nutrimentos en los árboles de teca en plantación es alta, comparada con un bosque tropical natural o con una plantación de pino tropical (Nwoboshi 1984). Según Kumar et ál. (2009) el 43\% de los nutrimentos en árboles de teca se encuentran en el tronco y la corteza y el $57 \%$ se encuentra en las hojas, ramas y partes reproductivas. Los nutrimentos que mayormente se acumulan en la biomasa aérea de la teca son K, Ca y N (Hase y Foelster 1983, Nwoboshi 1984, Hernández et ál. 1993, Negi et ál. 1995, Kumar et ál. 2009, Portuguez 2012). Estos nutrimentos han sido estudiados en Nigeria, India, Venezuela y Costa Rica y el orden de la abundancia de los mismos podría depender del tipo de suelo en el cual crecen, de tal manera que el $\mathrm{N}$ es el elemento mayormente absorbido en un suelo fértil y el $\mathrm{K}$ es el elemento más absorbido en suelos ligeramente ácidos de menor fertilidad. La acumulación de nutrimentos varía con la edad, ya que el $\mathrm{N}$ es el nutrimento más absorbido en edades jóvenes y el Ca el elemento más absorbido en edades superiores (Nwoboshi 1984, Kumar et ál. 2009, Portuguez 2012).

La cantidad de micronutrimentos absorbida en otras especies como coníferas sigue el orden $\mathrm{Mn}>\mathrm{Fe}>\mathrm{Zn}>\mathrm{B}>\mathrm{Cu}$ con concentraciones más altas en el tronco y las ramas, con excepción del Mn, que se encontró en mayores cantidades en las acículas (Torraca et ál. 1984, Saur et ál. 1992) y en plantaciones de Alnus acuminata con el siguiente orden $\mathrm{Fe}>\mathrm{Zn}>\mathrm{Cu}>\mathrm{Mn}>\mathrm{B}$ (Segura et ál. 2005). El orden de acumulación de micronutrimentos en la teca en Costa Rica fue $\mathrm{Fe}>\mathrm{Zn}>\mathrm{B}>\mathrm{Mn}>\mathrm{Cu}$ (Portuguez 2012). La acumulación total de micronutrimentos por árbol varía desde $1 \mathrm{~g}$ en el año ${ }^{-1}$ hasta $45 \mathrm{~g}$ en el año 17 (Portuguez 2012).

Según Blanco et ál. (2009) el manejo de un bosque puede afectar la forma en como los árboles usan los nutrimentos, por lo tanto el manejo de podas, raleos y corta final en teca debe asumir la búsqueda de un equilibrio del sistema para la conservación de los nutrimentos. De acuerdo con Martiarena et ál. (2010), aparte del suelo, el componente arbóreo de un sistema forestal es el de mayor acumulación de nutrimentos, por lo que es importante conservar los residuos de los raleos y de la cosecha final.

\section{MATERIALES Y MÉTODOS}

La zona de estudio se ubicó dentro de la cuenca del Canal de Panamá, entre las coordenadas geográficas $9^{\circ} 00^{\prime}$ y $9^{\circ} 10^{\prime}$ Norte, $79^{\circ} 45^{\prime}$ y $80^{\circ} 00^{\prime}$ Oeste. Los suelos son mayormente rojos, arcillosos y ácidos, clasificados como Ultisoles, en la zona de vida bosque húmedo tropical según la clasificación de Holdridge (TLBG 2006). La temperatura promedio anual es de $26,3^{\circ} \mathrm{C}$ y la precipitación promedio anual es de $2880 \mathrm{~mm}$ (TLBG 2006).

El sitio de muestreo consistió en parcelas permanentes de crecimiento que formaban parte de una red establecida previamente al estudio y clasificada según el incremento medio anual en volumen $\left(\mathrm{IMA}_{\mathrm{v}}\right)$. Las parcelas se clasificaron por rendimiento en bajo $\left(2,9-5,3 \mathrm{~m}^{3} \mathrm{ha}^{-1} \cdot \mathrm{año}^{-1}\right)$, medio $\left(5,4-9,3 \mathrm{~m}^{3} \mathrm{ha}^{-1} \cdot \mathrm{año}^{-1}\right)$ y alto crecimiento $(9,4-13,3$ $\mathrm{m}^{3}$ ha $\left.^{-1} \cdot \mathrm{año}^{-1}\right)$ respectivamente, categorías que corresponden a crecimientos muy bajos, bajos y medios a nivel Centroamericano y el Caribe, según información reportada por otros autores (FAO 1977, Keogh 1979, Vásquez y Ugalde 
1995, Vallejo 1996, Montero 1999, Ávila 2003, Vaides 2004). Se escogieron 16 parcelas dentro del grupo de alto crecimiento con el propósito de seleccionar 16 árboles representativos de cada una (uno por parcela). Los árboles seleccionados se utilizaron para cuantificar su biomasa aérea y determinar la concentración y acumulación de nutrimentos en los diferentes compartimentos de la biomasa aérea distribuidos en edades de 3 hasta 18 años. Los árboles fueron seccionados en 5 compartimentos: tronco (madera sin corteza), corteza, ramas primarias (RP), ramas secundarias (RS) y hojas, a edades de 3, 4, 5, 6, 7, 8, 9, 12, 14 y 18 años. En las edades 4, 5, 6, 7, 8, y 9 se cortaron 2 árboles por clase de edad para un total de 80 muestras de biomasa aérea. El trabajo de campo se realizó en agosto, con el fin de capturar el momento de mayor crecimiento vegetativo y por tanto una mayor representatividad de su estado nutricional. Los árboles seleccionados eran dominantes, sanos (follaje verde, copa completa, crecimiento en un solo eje o tronco), sin problemas fitosanitarios (enfermedades visibles como protuberancias, follaje descolorido, ataque de plagas como hormigas, comején o cualquier otro insecto), entre 10 y $30 \mathrm{~m}$ del borde de la parcela, de orilla de caminos, o de claros en la plantación. Como árbol representativo se seleccionó un individuo de posición sociológica dominante ya que el método del árbol promedio tiende a subestimar la biomasa seca total y el contenido de nutrimentos totales en un $27 \%$ respecto del método estratificado (Kaul et ál. 1979). Antes de talar cada árbol se limpió de hojas verdes caídas y se colocó una lona sobre la cual se dirigió el árbol. En lonas separadas extendidas sobre el suelo, se recogió la biomasa de cada una de las fracciones del árbol. Como ramas primarias se denominaron aquellas que salían del tronco, mientras que las secundarias fueron aquellas que salían de otra rama. Se pesó en fresco e in situ cada una de las fracciones totales por medio de una balanza con precisión de $0,2 \mathrm{~kg}$. Posteriormente, de cada fracción se obtuvo submuestras debidamente identificadas y se pesaron en una balanza con precisión de $0,025 \mathrm{~kg}$. De la muestra de follaje se obtuvo una submuestra conformada por 4 grupos de 10 hojas (40 en total por árbol) de diferentes partes del total y las hojas sin ningún criterio de selección. La muestra de ramas secundarias se hizo con 20 trozos pequeños tomados al azar del total y se envió al laboratorio, donde fueron molidos con un triturador con cuchillas. El tronco se partió en secciones consecutivas de $1 \mathrm{~m}$ y se procedió a quitarle la corteza. Para quitar la corteza se rayó el tronco longitudinalmente con la motosierra y la corteza se desprendió con una herramienta en forma de espátula. Para la toma de la muestra del tronco en segmentos de $25 \mathrm{~cm}$ con la ayuda de una motosierra y se recogió $1 \mathrm{~kg}$ de aserrín sobre una lona limpia. La muestra de corteza y ramas primarias se obtuvo al cortar con motosierra, todas las secciones para recoger luego, cerca de $1 \mathrm{~kg}$ de aserrín previamente homogeneizado para cada caso y colocado en bolsas plásticas. Antes de recoger el aserrín se aseguró de eliminar la contaminación con basuras o cualquier pedazo de material sin cortar.

El uso de la motosierra permitió obtener una muestra homogénea, ya que se usó material de todos los segmentos del tronco, además la cantidad de aserrín fue proporcional al tamaño de las partes cortadas. Las muestras se trasladaron al laboratorio de suelos y foliares del Centro de Investigaciones Agronómicas de la Universidad de Costa Rica (CIA). Los nutrimentos que se determinaron fueron: $\mathrm{N}, \mathrm{P}, \mathrm{Ca}, \mathrm{Mg}, \mathrm{K}$ y $\mathrm{S}$ en porcentaje, mientras que $\mathrm{Fe}, \mathrm{Cu}, \mathrm{Zn}, \mathrm{Mn}$ y $\mathrm{B}$ en mg. $\mathrm{kg}^{-1}$. El análisis de las muestras foliares se realizó a través de la metodología de digestión húmeda y se empleó el extracto para efectuar la lectura de concentración por espectrofotometría de emisión atómica con plasma para el $\mathrm{Ca}, \mathrm{Mg}$, $\mathrm{K}, \mathrm{P}, \mathrm{S}, \mathrm{B}, \mathrm{Al}, \mathrm{Zn}, \mathrm{Cu}, \mathrm{Mn}$, Mo y Fe y el $\mathrm{N}$ por el método Microkjeldahl (Henríquez et ál. 1995).

\section{Determinación de la Biomasa Aérea}

Se pesó la biomasa fresca (en campo) y se determinó la biomasa seca total por árbol (laboratorio). Se determinó en el laboratorio el peso seco de cada una de las muestras, colocándola en la estufa a $105^{\circ} \mathrm{C}$, lo que permitió conocer el 
contenido de humedad de cada una de estas; a partir del contenido de humedad de cada muestra fue posible determinar el peso anhidro de cada uno de los compartimentos de los árboles.

Se ajustó un modelo de predicción por regresión para la biomasa fresca y seca total por árbol, en función con la edad desde los 3 hasta los 18 años. También se ajustaron modelos matemáticos según la edad, desde los 3 hasta los 18 años para cada una de las fracciones de biomasa seca de tronco, rama primaria, rama secundaria, corteza y follaje.

\section{Determinación de la Concentración de Nutrimentos}

Para cada uno de los macro y micronutrimentos según cada fracción del árbol, se obtuvo el valor promedio, desviación estándar y el coeficiente de variación; se hizo una comparación de medias de concentración de nutrimentos en los compartimentos sin distinción de la edad (según Tukey $\alpha=0,05$ ).

\section{Acumulación de Nutrimentos en los Compartimentos de la Biomasa Aérea}

Se determinaron las ecuaciones de regresión por medio del programa Statistica 6,0 y se escogió la curva de mejor ajuste para calcular la acumulación total de todos los nutrimentos, según la edad y sin distinción de los compartimentos. Por acumulación total se entiende la sumatoria de todos los nutrimentos $(\mathrm{N}, \mathrm{P}, \mathrm{K}, \mathrm{Ca}$, $\mathrm{Mg}, \mathrm{S}, \mathrm{Fe}, \mathrm{Cu}, \mathrm{Mn}, \mathrm{Zn}, \mathrm{B}$ en kg.árbol ${ }^{-1}$ a una edad determinada). Posteriormente, se graficaron las curvas de acumulación de la biomasa aérea por compartimento, la cantidad relativa acumulada de cada nutrimento y la acumulación anual absoluta para cada compartimento según la edad.

Se confeccionaron ecuaciones para la estimación de la acumulación total de cada nutrimento a edades desde 3 hasta 18 años. Se calcularon las ecuaciones a cada edad y para cada nutrimento y se obtuvo la diferencia de acumulación entre años (Ej: año 4 menos año 3) para estimar la acumulación interanual (STATSOFT INC. 2001).

\section{RESULTADOS Y DISCUSIÓN}

\section{Producción de Biomasa Aérea}

La biomasa seca de los árboles de teca de 3 a 18 años varió desde los $34 \mathrm{~kg}$.árbol $^{-1}$ hasta los $1286 \mathrm{~kg} . a ́ r b o l^{-1}$ con un IMA $_{\text {Biomasa }}$ (Incremento Medio Anual en Biomasa $=$ peso.edad ${ }^{-1}$ ) de 71,4 kg.árbol ${ }^{-1}$.año ${ }^{-1}$ a la edad de 18 años. Los valores obtenidos en India por Negi et ál. (1995) fueron menores ya que a los 20 años la biomasa seca total fue de $181 \mathrm{~kg}^{2}$ árbol ${ }^{-1}$, con un índice de

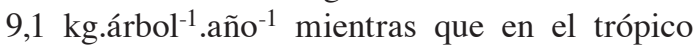
estacionalmente seco de Costa Rica Portuguez (2012) determinó un IMa ${ }_{\text {Biomasa }}=41 \mathrm{~kg}$ por árbol a la edad de 17,1 años. Estas variaciones se explican debido a que la biomasa de los árboles de teca está en función de factores ambientales, de la fertilidad del suelo, del material genético y del manejo de la plantación. Según Thiele (2008), el crecimiento de la teca se ve favorecido en los sitios planos de origen aluvial, coluvial o fluvio-volcánico, sin problemas de drenaje, con no más de 3 meses secos, profundidad efectiva mayor a $64 \mathrm{~cm}$ y una precipitación mayor de $2000 \mathrm{~mm}$ anuales.

La biomasa seca promedio del tronco a la edad de 1 a 18 años representó el 59,6\% (C.V. 5\%) de la biomasa aérea seca total, mientras que las ramas primarias, ramas secundarias, corteza y hojas representaron el $16,6 \%, 6,5 \%, 9,4 \%$ y $7,9 \%$ del total, respectivamente. La biomasa del tronco y la corteza representó el 69\%, valor similar a los obtenidos por Portuguez (2012) y por Mbaekwe y Mackenzie (2008) quienes determinaron que la biomasa seca del tronco con corteza varió desde 69 a 76\% respecto del total. La biomasa seca por árbol podría ser determinada con la ecuación $y=22,211 \mathrm{e}^{0,2094 \mathrm{x}}$, donde " $\mathrm{x}$ " es la edad y el $\mathrm{R}^{2}=0,94$ (Figura 1). La biomasa fresca por árbol podría ser determinada con la ecuación y=62,093 * $\mathrm{e}^{0,1947 \mathrm{x}}$, donde " $\mathrm{x}$ " es la edad y el $\mathrm{R}^{2}=0,94$.

Se estableció una función de regresión para la determinación de la biomasa seca de cada uno de los compartimentos del árbol de teca desde 3 hasta 18 años: biomasa del tronco 


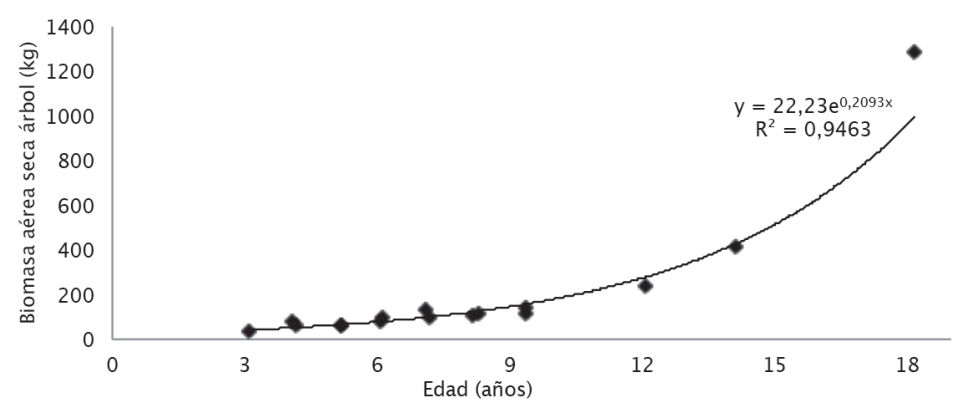

Fig. 1. Curva de biomasa seca total por árbol de teca entre 3 y 18 años, en la zona del Canal de Panamá.

$=13,212 \mathrm{e}^{0,2096 x}\left(\mathrm{R}^{2}=0,95\right)$, biomasa de ramas primarias $=3,6721 \mathrm{e}^{0,2074 \mathrm{x}}\left(\mathrm{R}^{2}=0,85\right)$, biomasa de ramas secundarias $=0,729 \mathrm{e}^{0,2823 \mathrm{x}}\left(\mathrm{R}^{2}=0,84\right)$, biomasa de corteza $=2,2318 \mathrm{e}^{0,1991 x}\left(\mathrm{R}^{2}=0,97\right) \mathrm{y}$ la biomasa de las hojas $=2,7035 \mathrm{e}^{0,148 \mathrm{x}}\left(\mathrm{R}^{2}=0,81\right)$. Todas las funciones ajustadas mostraron una tendencia hacia el aumento en forma exponencial con la edad de los árboles, en vez de ajustarse a un modelo sigmoideo, aspecto que indica que los árboles todavía mantienen el ritmo de crecimiento rápido según criterios desarrollados por Vimal et ál. (2003) (Figura 2). Portuguez (2012), Negi et ál. (1995) y Salas e Infante (2006) determinaron ecuaciones para biomasa con altos valores de $\mathrm{R}^{2}$ pertinentes a los sitios por ellos estudiados y que podrían ser extrapoladas únicamente bajo condiciones ambientales y de material genético similar. 

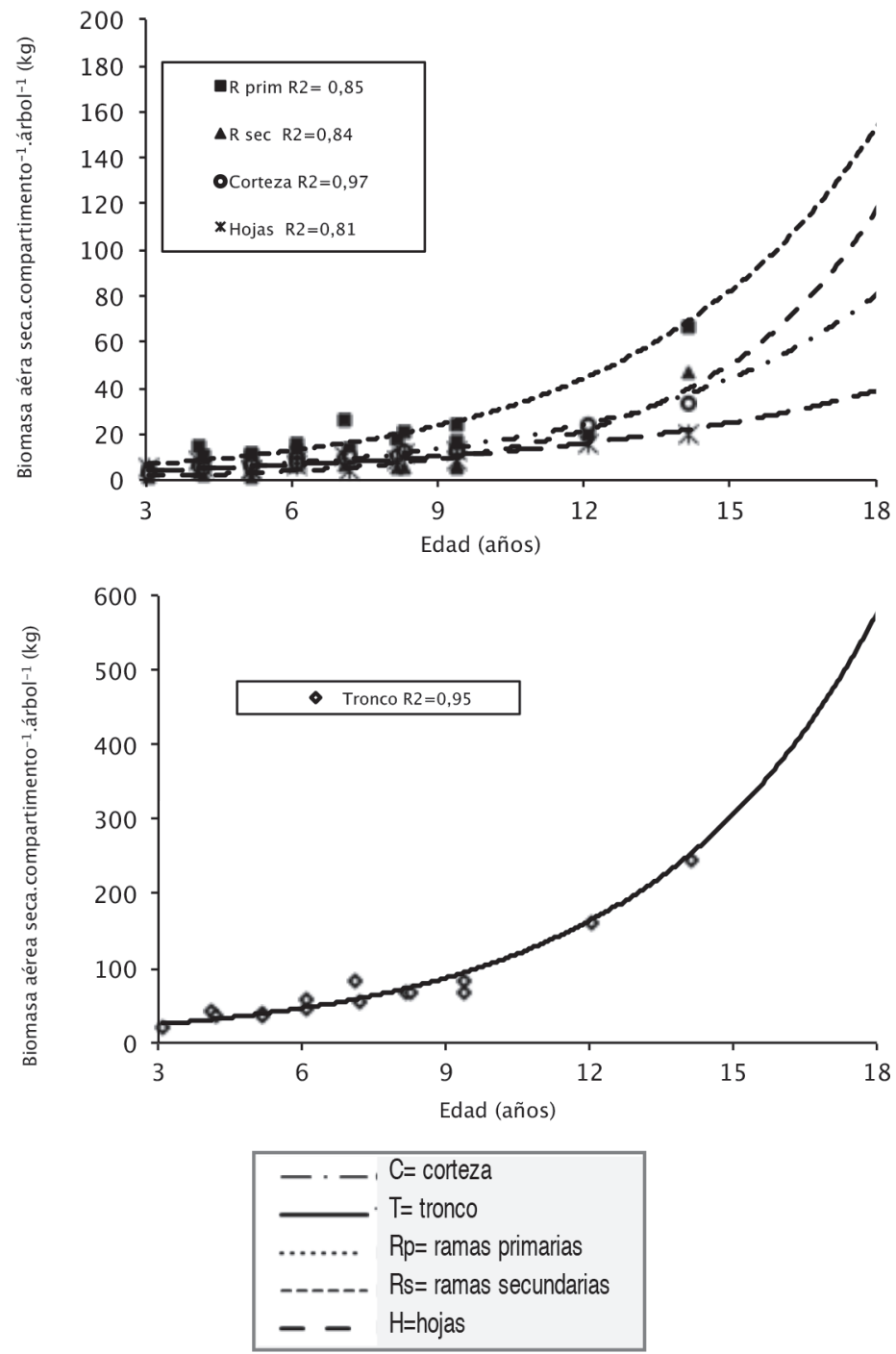

Fig. 2. Curvas de biomasa seca por compartimento y por árbol de teca entre 3 y 18 años, en la zona del Canal de Panamá.

\section{Concentración de Nutrimentos}

El compartimento de la biomasa aérea con la mayor concentración de macronutrimentos es decir, la sumatoria de concentraciones (Cuadro 1), fue el follaje con $4,66 \%$, seguido por la corteza con $4,17 \%$ y la menor cantidad de macronutrimentos se encontró en el tronco con $0,72 \%$.
En general, el orden de la concentración individual de los macronutrimentos en este estudio (Cuadros 1 y 2) concuerda con el orden encontrado en otros trabajos desarrollados en Nigeria (Nwoboshi 1984), India (Negi 1990) y Costa Rica (Montero y Kanninen 2006, Portuguez 2012). Podría haber un efecto de sitio y de edad de los árboles sobre la concentración 
Cuadro 1. Concentración de macronutrimentos en los compartimentos de la biomasa de teca entre 3 a 18 años, en la zona del Canal de Panamá.

\begin{tabular}{|c|c|c|c|c|c|c|c|c|c|c|c|c|c|}
\hline \multirow{2}{*}{ Compartimento } & \multicolumn{2}{|c|}{$\mathrm{N}$} & \multicolumn{2}{|c|}{$\mathrm{P}$} & \multicolumn{2}{|c|}{$\mathrm{K}$} & \multicolumn{2}{|c|}{$\mathrm{Ca}$} & \multicolumn{2}{|c|}{$\mathrm{Mg}$} & \multicolumn{2}{|c|}{$\mathrm{S}$} & \multirow{2}{*}{$\begin{array}{c}\text { Total } \\
\% \\
\end{array}$} \\
\hline & $\%$ & \pm D.E & $\%$ & \pm D.E & $\%$ & \pm D.E & $\%$ & \pm D.E & $\%$ & \pm D.E & $\%$ & \pm D.E & \\
\hline Corteza & 0,69 & 0,07 & 0,07 & 0,02 & 1,07 & 0,30 & 2,01 & 0,42 & 0,26 & 0,11 & 0,07 & 0,01 & 4,17 \\
\hline Hojas & 1,98 & 0,19 & 0,13 & 0,03 & 1,02 & 0,20 & 1,16 & 0,42 & 0,25 & 0,08 & 0,12 & 0,01 & 4,66 \\
\hline $\mathrm{RP}$ & 0,45 & 0,07 & 0,04 & 0,02 & 0,54 & 0,17 & 0,74 & 0,22 & 0,13 & 0,05 & 0,05 & 0,02 & 1,95 \\
\hline $\mathrm{RS}$ & 0,54 & 0,07 & 0,05 & 0,02 & 0,73 & 0,20 & 0,91 & 0,25 & 0,16 & 0,04 & 0,06 & 0,01 & 2,45 \\
\hline Tronco & 0,27 & 0,04 & 0,05 & 0,03 & 0,19 & 0,09 & 0,11 & 0,03 & 0,08 & 0,02 & 0,02 & 0,01 & 0,72 \\
\hline
\end{tabular}

$\mathrm{n}=16$ (corteza, hojas, ramas primarias, ramas secundarias, tronco), $\mathrm{n}=7$ (flores).

Cuadro 2. Concentración de micronutrimentos en los compartimentos de la biomasa de teca entre 3 a 18 años, en la zona del Canal de Panamá.

\begin{tabular}{|c|c|c|c|c|c|c|c|c|c|c|c|}
\hline \multirow{2}{*}{ Compartimento } & \multicolumn{2}{|r|}{$\mathrm{Fe}$} & \multicolumn{2}{|r|}{$\mathrm{Cu}$} & \multicolumn{2}{|r|}{$\mathrm{Mn}$} & \multicolumn{2}{|r|}{$\mathrm{Zn}$} & \multicolumn{2}{|r|}{ B } & Total \\
\hline & $\mathrm{mg} \cdot \mathrm{kg}^{-1}$ & \pm D.E & $\mathrm{mg} \cdot \mathrm{kg}^{-1}$ & \pm D.E & $\mathrm{mg} \cdot \mathrm{kg}^{-1}$ & \pm D.E & $\mathrm{mg} \cdot \mathrm{kg}^{-1}$ & \pm D.E & $\mathrm{mg} \cdot \mathrm{kg}^{-1}$ & \pm D.E & $\mathrm{mg} \cdot \mathrm{kg}^{-1}$ \\
\hline Corteza & 767,1 & 736,0 & 6,6 & 5,7 & 60,4 & 40,4 & 50,2 & 13,4 & 31,9 & 5,3 & 916,2 \\
\hline Hojas & 138,6 & 92,6 & 10,3 & 2,6 & 41,1 & 23,5 & 30,0 & 8,9 & 17,8 & 2,9 & 237,8 \\
\hline $\mathrm{RP}$ & 147,6 & 126,1 & 3,6 & 1,0 & 19,3 & 18,8 & 26,0 & 17,7 & 10,4 & 2,6 & 206,9 \\
\hline $\mathrm{RS}$ & 74,6 & 34,6 & 6,6 & 1,5 & 18,9 & 9,3 & 17,1 & 7,7 & 11,8 & 2,6 & 129 \\
\hline Tronco & 125,0 & 102,0 & 2,4 & 2,0 & 2,6 & 2,2 & 20,2 & 11,0 & 3,1 & 1,6 & 153,3 \\
\hline
\end{tabular}

y eso se nota en la variación que se presenta en el K foliar, que en Panamá fue de $1,02 \%$ (este estudio, Ultisoles), en Nigeria tenía valores de $2,39 \%$ (suelos fértiles) a la edad de 15 años y en Costa Rica $0,84 \%$ en suelos clasificados como Molisoles, Alfisoles y Ultisoles entre 1 y 17 años (Portuguez 2012).

El compartimento de la biomasa aérea con la mayor concentración (sumatoria de concentraciones) de micronutrimentos fue la corteza con $916 \mathrm{mg} \cdot \mathrm{kg}^{-1}$, mientras que el compartimento con la menor cantidad de micronutrimentos fue el tronco con 153 mg. $\mathrm{kg}^{-1}$ (Figura 3).

Los macronutrimentos de mayor concentración individual según los compartimentos de la biomasa fueron el Ca $(2,01 \%)$ en la corteza y el N (1,98\%) en las hojas. Los micronutrimentos de mayor concentración individual según el compartimento de biomasa fueron el Fe (767 mg. $\left.\mathrm{kg}^{-1}\right)$, Mn (60 mg. $\left.\mathrm{kg}^{-1}\right)$ y Zn $\left(50 \mathrm{mg} \cdot \mathrm{kg}^{-1}\right)$, todos en la corteza. Se registraron diferencias significativas de concentración entre algunos de los compartimentos de la biomasa aérea (Cuadro 3).

La letra $\mathrm{A}$ en el Cuadro 3 representa un valor promedio de concentración menor y las letras subsecuentes representan valores de concentración mayores. Puede observarse que las menores diferencias de concentración entre compartimentos de la biomasa aérea ocurrieron para los elementos P, Fe y Zn. 


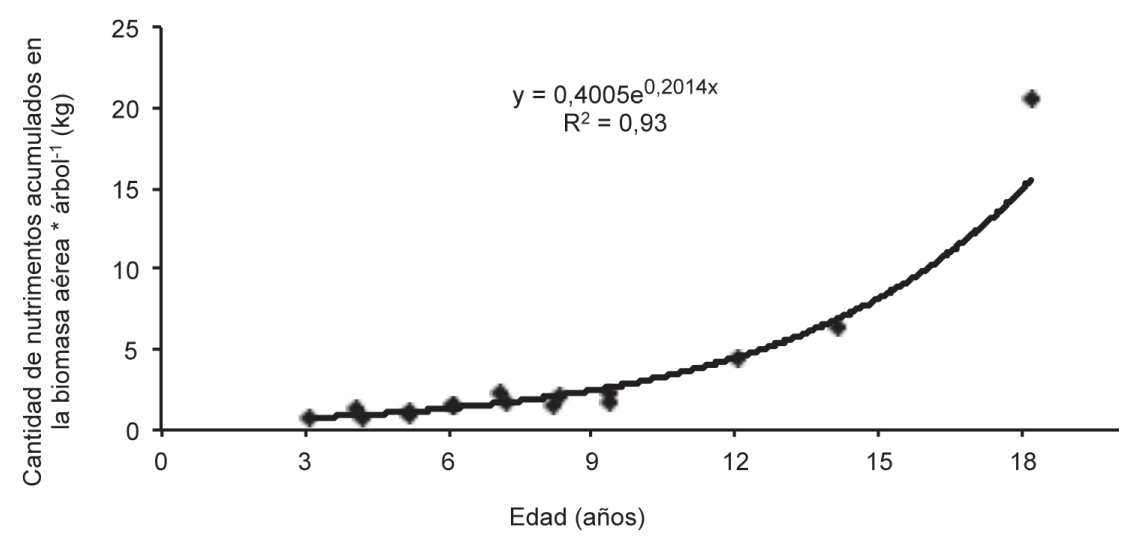

Fig. 3. Curva de acumulación total de nutrimentos por árbol de teca según la edad, en la cuenca del Canal de Panamá.

Cuadro 3. Comparación entre las medias de concentración de macro y micronutrimentos (Tukey $(\alpha=0,05))$ según el compartimento de biomasa en plantaciones de teca en la zona del Canal de Panamá.

\begin{tabular}{l|cccccc|ccccc}
\hline \multirow{2}{*}{ Compartimento } & \multicolumn{7}{|c|}{ Macronutrimentos } & \multicolumn{5}{c}{ Micronutrimentos } \\
\cline { 2 - 13 } & N & P & K & Ca & Mg & S & Fe & Cu & Mn & Zn & B \\
\hline Corteza & C & A & C & C & C & C & B & B & C & B & D \\
Hojas & E & B & C & D & C & E & A & C & BC & A & C \\
RP & B & A & B & B & AB & B & A & AB & AB & A & B \\
RS & B & A & B & D & B & BC & A & B & AB & A & B \\
Tronco & A & A & A & A & A & A & A & A & A & A & A \\
\hline
\end{tabular}

Letras diferentes entre las casillas de una misma columna del Cuadro 3 representan diferencias estadísticamente significativas (probabilidad de un 95\%).

\section{Acumulación Total de Nutrimentos por Árbol/Edad}

La acumulación total de nutrimentos por árbol en la teca según edad se puede explicar con la ecuación $\mathrm{y}=0,4005 * \mathrm{e}^{0,2014 \mathrm{X}}$, donde " $x$ " = edad del árbol, con un $\mathrm{R}^{2}$ de 0,93 (Figura 3). Los macronutrimentos representan el $98,6 \%$ del total de nutrimentos acumulados y los micronutrimentos corresponden solamente al 1,4\%.

Las curvas de acumulación del $\mathrm{N}, \mathrm{Ca}$, $\mathrm{Mg}, \mathrm{S}, \mathrm{K}$ y en menor grado el $\mathrm{P}$ tienen tendencias crecientes para los distintos compartimentos de la biomasa de la teca (Figura 4), ya que se encuentran definidos principalmente por los incrementos de la biomasa. El 58\% del N acumulado se encuentra mayormente en el tronco y las hojas, el Mg, P y S (40\%, 61\%, 32\% respectivamente) se encuentran mayormente en el tronco, mientras que el $68 \%$ del $\mathrm{K}$ absorbido se encuentra en tronco, ramas primarias y corteza (Figura 4). Por otro lado el $33 \%$ del Ca acumulado se encuentra en mayor cantidad en la corteza.

La acumulación de nutrimentos reportada para teca en Nigeria varía respecto de la acumulación en Panamá, ya que un árbol de 15 años en Nigeria absorbe 2,86 $\mathrm{kg}$ de K, 2,14 $\mathrm{kg}$ de Ca, 1,71 $\mathrm{kg}$ de $\mathrm{N}, 0,43 \mathrm{~kg}$ de $\mathrm{P}$ y $0,36 \mathrm{~kg}$ de $\mathrm{Mg}$ (Nwoboshi 

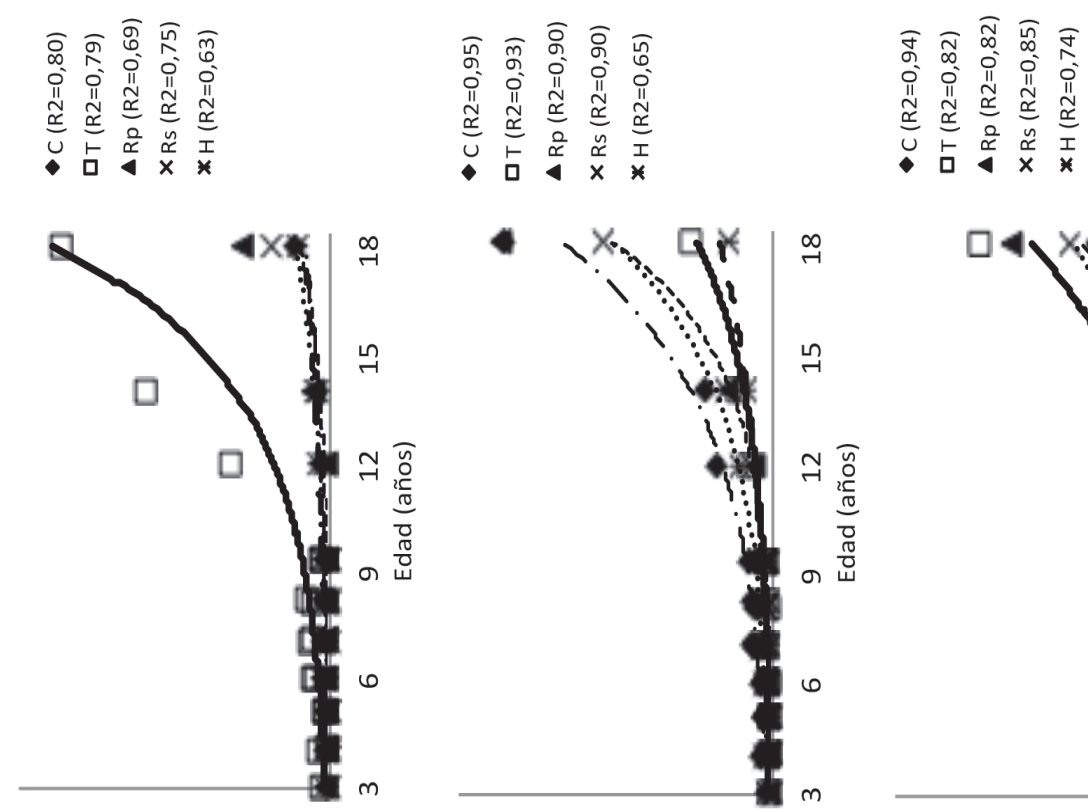

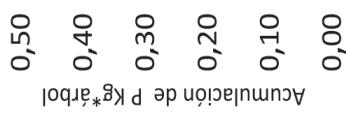

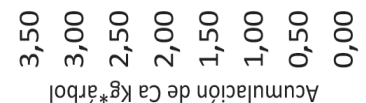
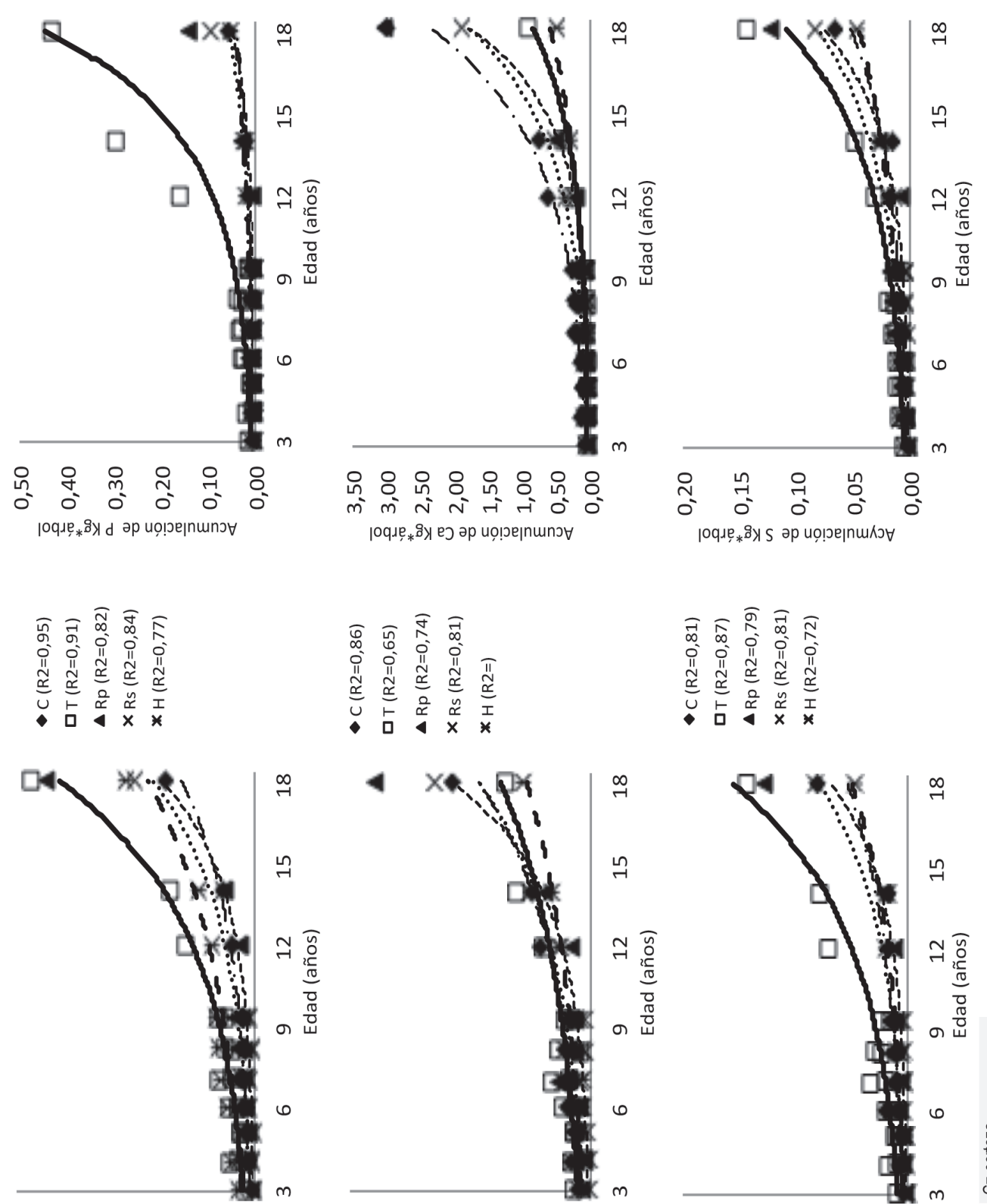

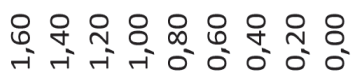

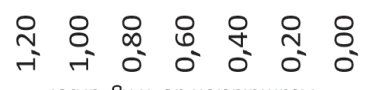

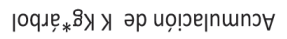

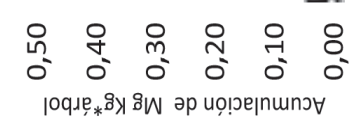

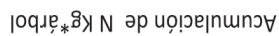

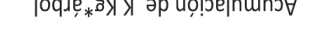


1984), mientras que un árbol de 14 años en Panamá (en esta investigación) acumula $2,49 \mathrm{~kg}$ de $\mathrm{Ca}, 1,57 \mathrm{~kg}$ de $\mathrm{N}, 1,40 \mathrm{~kg}$ de $\mathrm{K}$ y $0,38 \mathrm{~kg}$ de $\mathrm{P}$ y $0,38 \mathrm{~kg}$ de $\mathrm{Mg}$. Por tanto, un árbol de teca de 15 años en Nigeria absorbe más N, P y K que un árbol de 14 años en Panamá, que absorbe más Ca y Mg que uno de Nigeria. Un árbol de teca de 14 años en Guanacaste, Costa Rica acumula $2,7 \mathrm{~kg}$ de Ca, 2 kg de N y 0,9 kg de K (Portuguez 2012), es decir más que uno en Panamá, probablemente debido a que en Guanacaste hay mayor disponibilidad de estos elementos en el suelo.

En general, las curvas de acumulación de los micronutrimentos (Figura 5) son crecientes en relación con el incremento en edad, principalmente a partir del año 10 . El Fe, el Cu y el Zn, son los microelementos mayormente acumulados en el tronco $(47 \%, 43 \%$ y $52 \%$ respectivamente), el $31 \%$ del Mn es mayormente acumulado en la corteza y las ramas primarias y el $\mathrm{B}$ es acumulado en mayor parte en la corteza, tronco y ramas primarias $(31 \%, 22 \%, 22 \%$ respectivamente).

La proporción relativa de la acumulación total de Ca por árbol (Figura 6) tiene la tendencia a aumentar después del año 7 hasta el 18 (formación de tejido leñoso) mientras que la proporción relativa de la acumulación total de $\mathrm{K}$ tiene la tendencia a disminuir con la edad a partir del año 7 , probablemente debido a falta de espacio para el crecimiento de la copa donde el elemento se utiliza para regular procesos osmóticos. La proporción relativa total de acumulación de $\mathrm{S}$, Mg y P se mantiene estable desde los 3 hasta los 18 años. Estas tendencias también se observaron en los datos desarrollados por Nwoboshi (1984) en Nigeria, sin embargo, las diferencias están en los valores absolutos de acumulación, los cuales dependen de la disponibilidad para ser acumulados. En relación con los micronutrimentos no se encontró ninguna tendencia en la tasa de acumulación con respecto a la edad (Figura 6). 


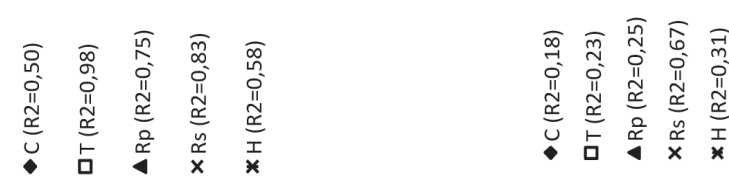

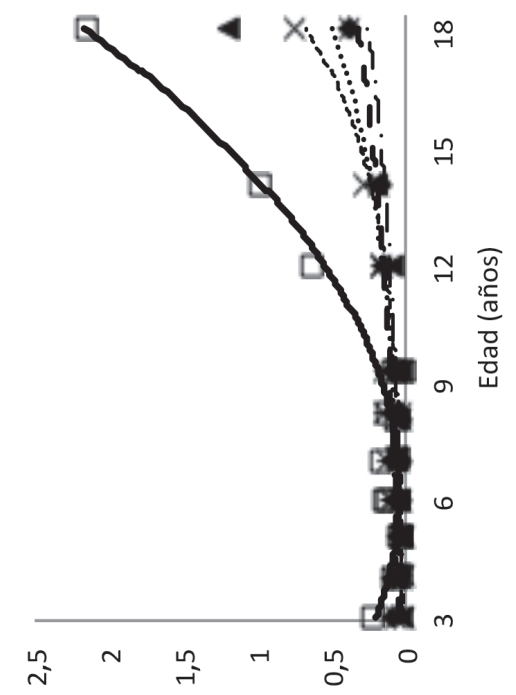

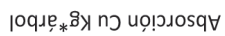
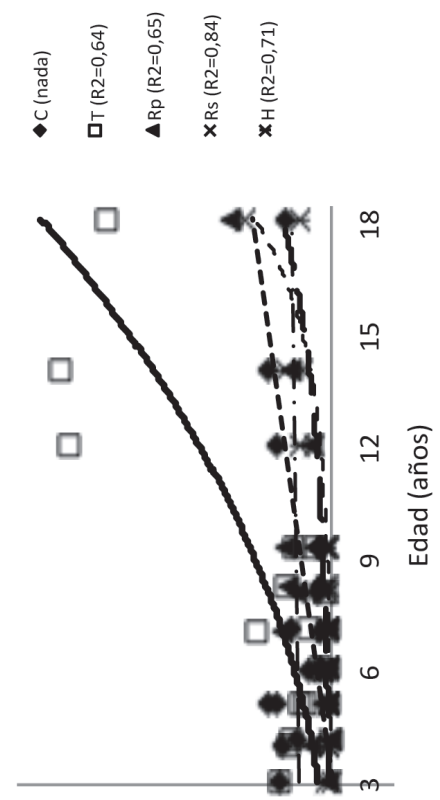

오 아 유 우 0 우

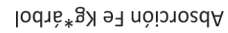
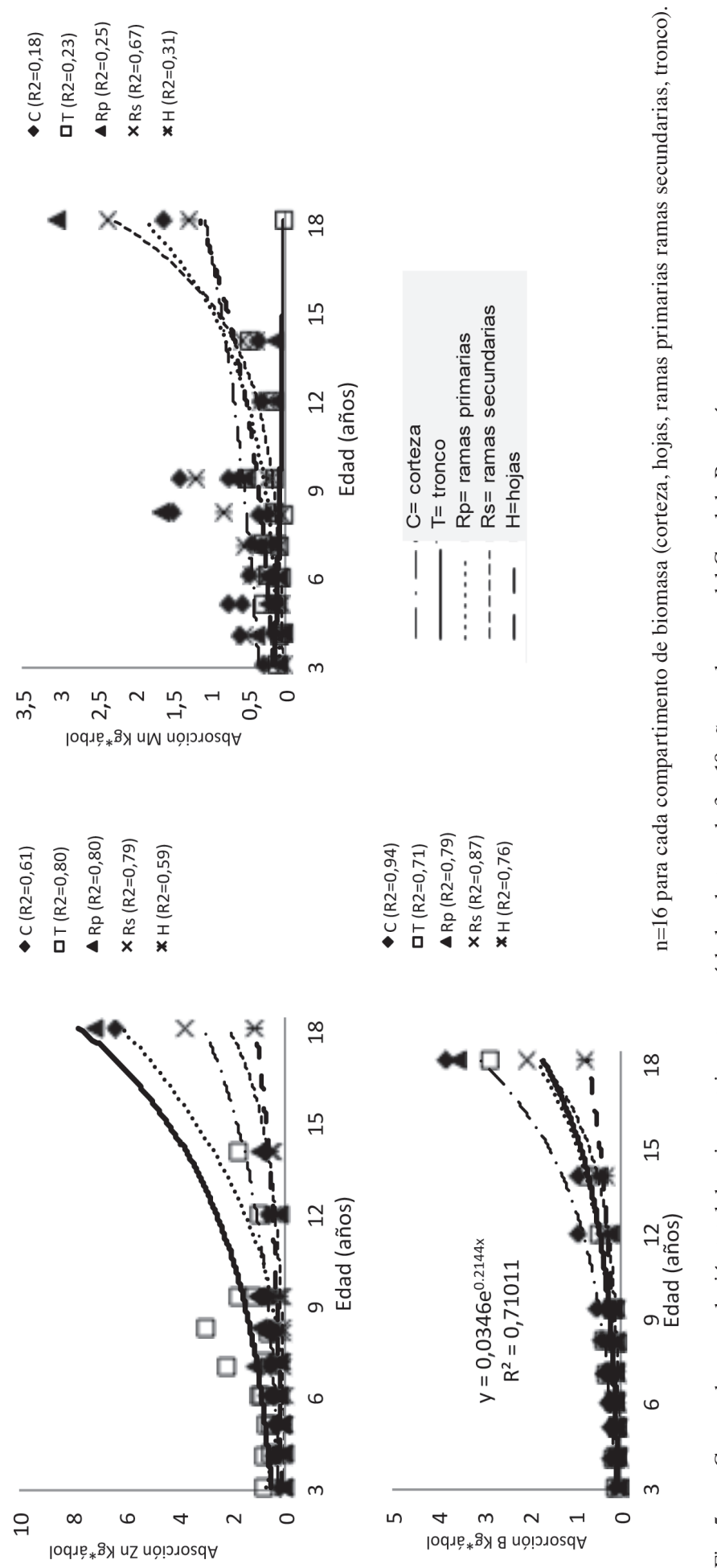

Agronomía Costarricense 39(3): 117-136. ISSN:0377-9424 / 2015 

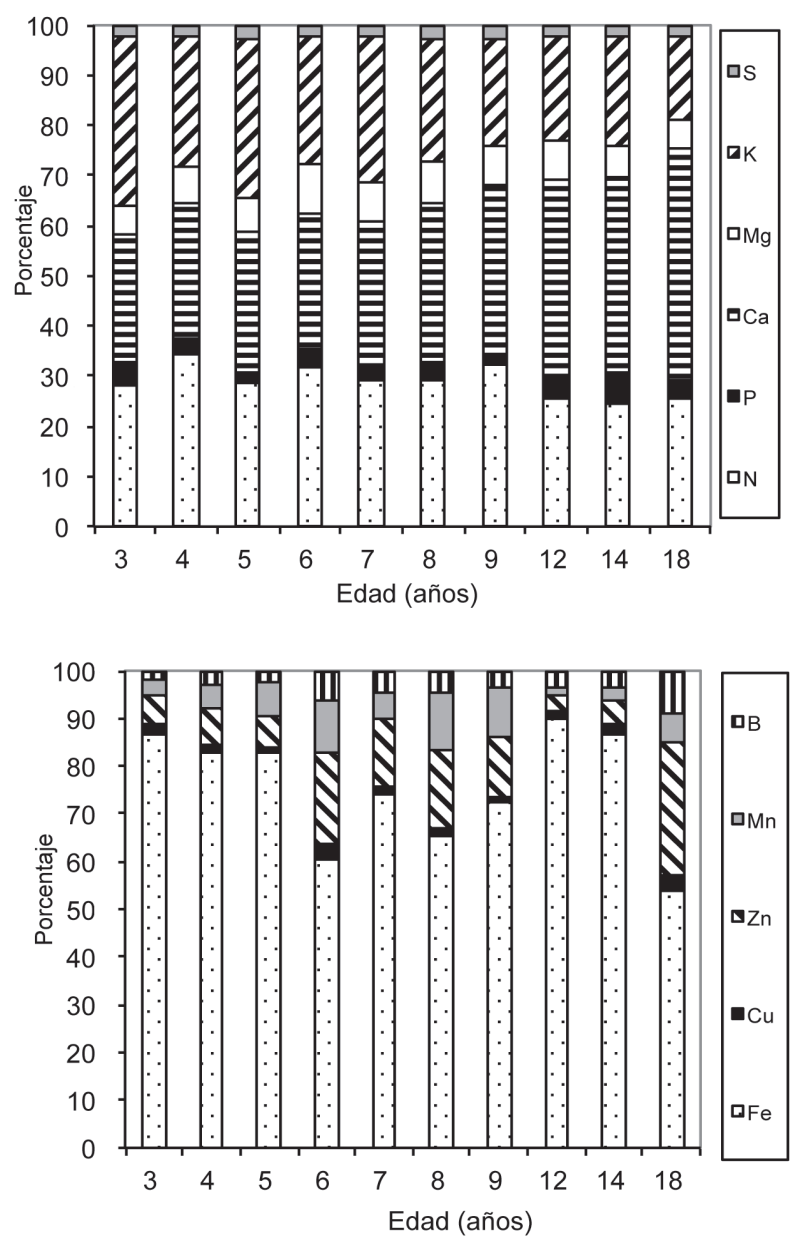

Fig. 6. Acumulación relativa de cada macro y micronutrimento por árbol según la edad, en plantaciones de teca de la zona del Canal de Panamá.

Para cada uno de los macronutrimentos acumulados fue posible ajustar una función de estimación en cada compartimento de biomasa, con un coeficiente de determinación mayor de 0,6 (Cuadro 4). Las mejores ecuaciones de estimación para cada nutrimento se obtuvieron para la corteza seguida del tronco, mientras que las de menor ajuste fueron para los macronutrimentos en la biomasa foliar. 


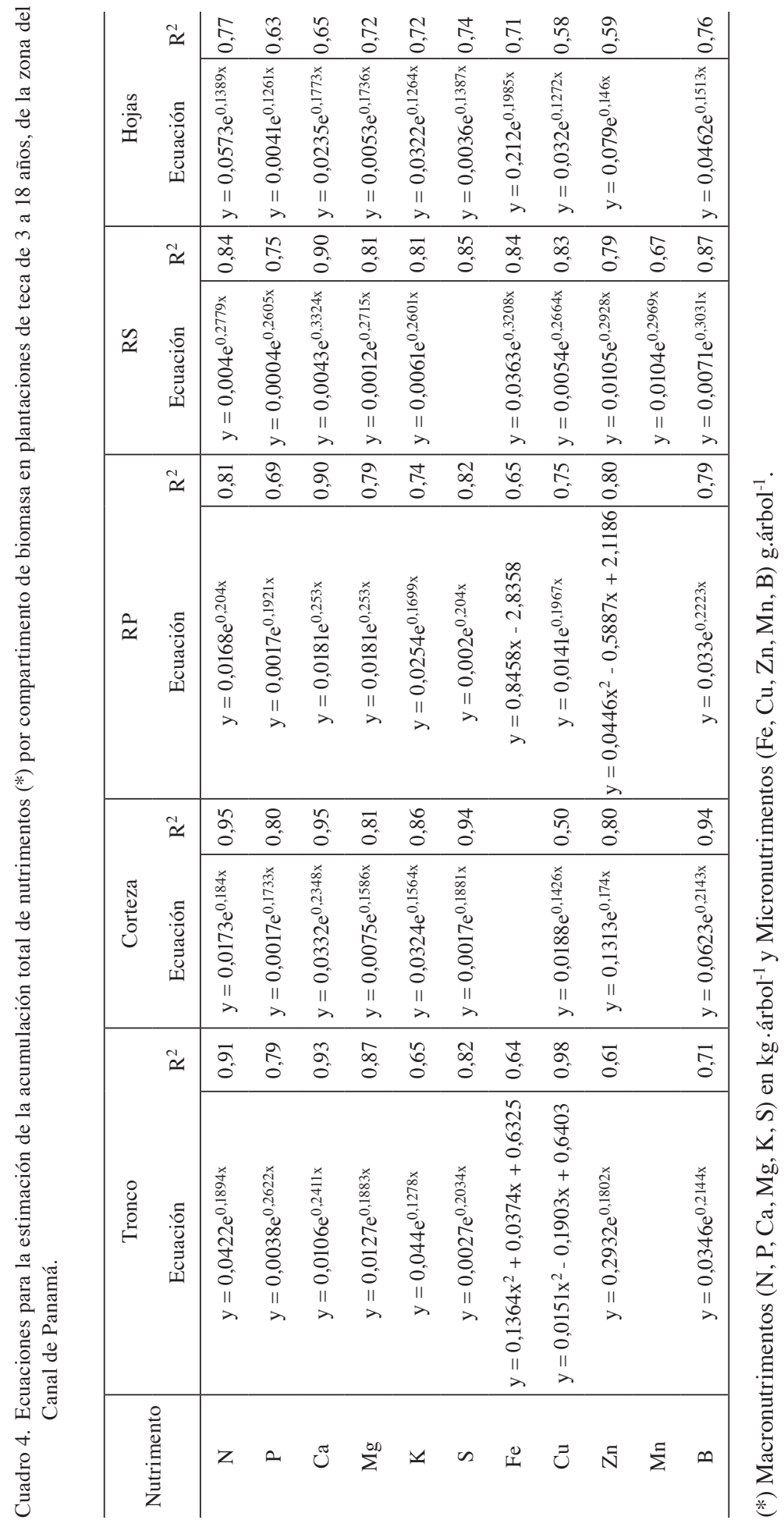


Las funciones de acumulación de micronutrimentos en los compartimentos de la biomasa aérea registraron en general un menor valor de ajuste $\left(\mathrm{R}^{2}\right)$ que con las funciones de los macronutrimentos. La acumulación del $\mathrm{Fe}, \mathrm{Cu}, \mathrm{Zn}$ y B fue explicada por la edad en todos los compartimentos de biomasa estudiados, mientras que la acumulación de Mn solo fue explicada satisfactoriamente en la biomasa de las ramas secundarias.

Se determinó también, ecuaciones para la estimación de la acumulación total por árbol para cada uno de los nutrimentos desde el año 3 hasta el año 18 (Cuadro 5). Para todos los macronutrimentos los coeficientes de determinación fueron mayores al 63\%, mientras que en el caso de los micronutrimentos los valores de $\mathrm{R}^{2}$ fueron superiores a $50 \%$ de explicación de la variación. Con estas funciones de predicción se puede calcular la acumulación de nutrimentos por árbol.año $0^{-1}$ para cada macro y micronutrimento y así se estimó que la acumulación total de nutrimentos fue de
$15,59 \mathrm{~kg}^{\text {árbol }}{ }^{-1}$ hasta la edad de 18 años $(7,32 \mathrm{~kg}$ de Ca, 3,9 kg de N, 2,66 kg de K, 1,03 kg de Mg y $0,69 \mathrm{~kg}$ de P). La cantidad de nutrimentos acumulada y reportada en otros estudios como en India (Kumar 2009) a la edad de 18,5 años fue mucho menor con 2,21 kg.árbol ${ }^{-1}(0,80 \mathrm{~kg}$ de N, $0,77 \mathrm{~kg}$ de Ca, 0,38 kg de K, 0,18 kg de Mg y 0,08 kg de P) comparada con los resultados de este estudio. Esta diferencia podría ser explicada en parte por la baja fertilidad de los suelos, la menor precipitación y menor tasa de crecimiento en general, así como por la alta densidad inicial de las plantaciones en India (1700 árboles por hectárea). La teca, es una especie exigente de suelos fértiles y la disponibilidad de nutrimentos dependerá de las reservas en el suelo (Vimal et ál. 2003), la velocidad del ciclo de cada nutrimento (Drechsel y Zech 1994), la distribución de los nutrimentos en los compartimentos del árbol (Nwoboshi 1984) y el suministro adecuado de nutrimentos por medio de los programas de fertilización.

Cuadro 5. Funciones para la estimación de la acumulación total de cada nutrimento por árbol en teca de 3 a 18 años.

\begin{tabular}{c|c|c|c}
\hline \multicolumn{2}{c|}{ Variable } & Ecuación & $\mathrm{R}^{2}$ \\
\hline \multirow{2}{*}{$\begin{array}{c}\text { Macro- } \\
\text { nutrimentos }\end{array}$} & $\mathrm{N}$ & $\mathrm{y}=0,1281 \mathrm{e}^{0,1897 \mathrm{x}}$ & 0,96 \\
$\mathrm{P}$ & $\mathrm{y}=0,0111 \mathrm{e}^{0,2295 \mathrm{x}}$ & 0,89 \\
$\mathrm{Ca}$ & $\mathrm{y}=0,0871 \mathrm{e}^{0,2462 \mathrm{x}}$ & 0,96 \\
$\mathrm{Mg}$ & $\mathrm{y}=0,0304 \mathrm{e}^{0,1957 \mathrm{x}}$ & 0,99 \\
$\mathrm{~K}$ & $\mathrm{y}=0,195782+\mathrm{e}^{(-3,2874+(0,244172 * \mathrm{X})}$ & 0,98 \\
\hline \multirow{3}{*}{$\begin{array}{c}\text { Micro- } \\
\text { nutrimentos }\end{array}$} & $\mathrm{S}$ & $\mathrm{y}=0,01 \mathrm{e}^{0,1988 \mathrm{x}}$ & 0,72 \\
& $\mathrm{Fe}$ & $\mathrm{y}=6,5956 \mathrm{e}^{0,1449 \mathrm{x}}$ & 0,82 \\
& $\mathrm{Cu}$ & $\mathrm{y}=0,0964 \mathrm{e}^{0,1983 \mathrm{x}}$ & 0,77 \\
& $\mathrm{Zn}$ & $\mathrm{y}=0,595 \mathrm{e}^{0,1881 \mathrm{x}}$ & 0,65 \\
\end{tabular}

(x) edad del árbol.

La necesidad anual de $\mathrm{Ca}$ y $\mathrm{N}$ por la teca es creciente (Figura 7) y los datos generados por las funciones de biomasa podrían utilizarse para la aplicación adecuada de fertilizantes bajo las condiciones de sitio de este estudio y tomando en cuenta la eficiencia de los mismos en suelos rojos y ácidos.

Los microelementos de mayor acumulación anual fueron el $\mathrm{Fe}$ y el $\mathrm{Zn}$, mientras que el de menor acumulación fue el $\mathrm{Cu}$. Portuguez 

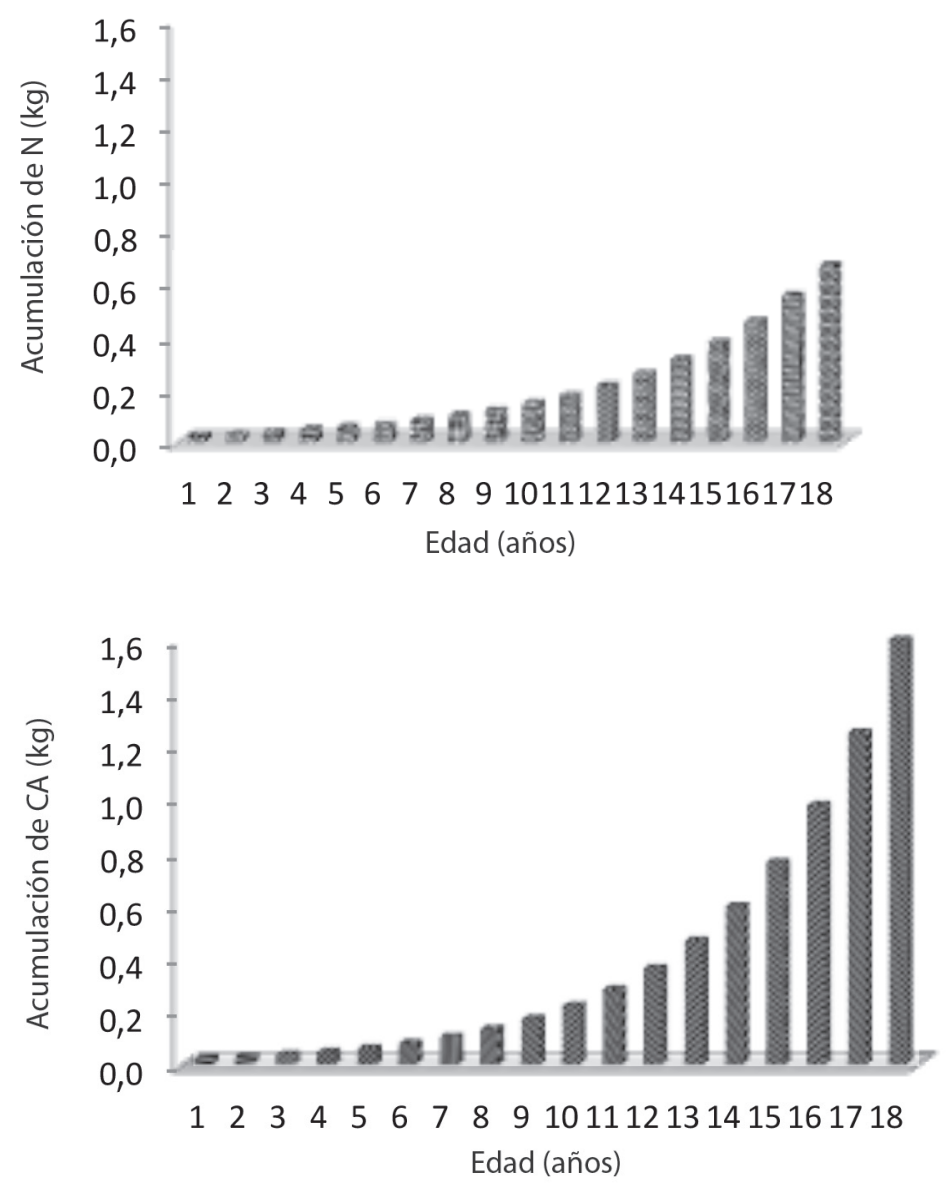

Fig. 7. Acumulación anual de Ca y N en árboles de teca en la Cuenca del Canal de Panamá.

(2012) en Costa Rica, determinó el siguiente orden de acumulación $\mathrm{Fe}, \mathrm{Zn}, \mathrm{B}, \mathrm{Mn}$ y Cu, el cual concuerda con los resultados de este estudio. El rango de acumulación anual del Fe calculado con la función fue desde $1,2 \mathrm{~g}$ en el año 3 hasta $12 \mathrm{~g}$ en el año $18 \mathrm{y}$ el rango del $\mathrm{Cu}$ fue de $0,03 \mathrm{~g}$ hasta los 0,60 g en el mismo período. La mayor parte, si no toda, de la cantidad acumulada de estos elementos, la suple el ecosistema, sea como elementos disponibles por meteorización del suelo, circulación o por adición de algunos de ellos vía precipitación pluvial o seca.
Actualmente se busca una reducción en el turno de corta de la teca, por medio del uso de tierras más fértiles, mejor preparación del suelo, uso de material genético de superior calidad, una mayor tasa de crecimiento y un mantenimiento adecuado, lo que sin duda lleva al uso de una tasa mayor de extracción de nutrimentos. Lo anterior debe servir para guiar la investigación hacia la búsqueda de genotipos de mayor eficiencia en el uso de los nutrimentos y genotipos con la capacidad de crecer bien en suelos de menor fertilidad, así como a la gestión de prácticas para la conservación de nutrimentos. 


\section{CONCLUSIONES}

\section{Biomasa Total Seca}

La biomasa seca de los árboles de teca de 3 a 18 años varió desde los $34 \mathrm{~kg}$.árbol ${ }^{-1}$ hasta los $1286 \mathrm{~kg}$.árbol ${ }^{-1}$, con un $\mathrm{IMA}_{\text {Biomasa }}\left(\right.$ peso edad ${ }^{-1}$ ) de 71,4 kg.árbol ${ }^{-1}$.año ${ }^{-1}$ a la edad de 18 años. La biomasa seca promedio del tronco representó el $59,6 \%$ de la biomasa seca total, mientras que las ramas primarias el $16,6 \%$, las ramas secundarias el $6,5 \%$, la corteza el $9,4 \%$ y las hojas el $7,9 \%$.

\section{Concentración de Nutrimentos en los Compartimentos de la Biomasa Aérea}

El compartimento de biomasa con la mayor concentración de macronutrimentos total fue el follaje con $4,66 \%$, seguido por la corteza con $4,17 \%$, mientras que el compartimento con la mayor cantidad de micronutrimentos total fue la corteza con 916 mg.kg ${ }^{-1}$. El compartimento con la menor concentración de macro y micronutrimentos total fue el tronco con $0,72 \%$ y 153 mg.kg ${ }^{-1}$, respectivamente. Los macronutrimentos de mayor concentración individual según compartimento de biomasa fueron el $\mathrm{Ca}(2,01 \%)$ en la corteza y el $\mathrm{N}(1,98 \%)$ en las hojas. Los micronutrimentos de mayor concentración individual fueron el Fe (767 mg. $\left.\mathrm{kg}^{-1}\right)$, Mn (60 mg. $\left.\mathrm{kg}^{-1}\right)$ y Zn (50 mg. $\left.\mathrm{kg}^{-1}\right)$, todos en la corteza.

\section{Acumulación de Nutrimentos en la Biomasa Aérea}

La acumulación de nutrimentos a la edad de 18 años asociada a un rendimiento de 1286 kg.árbol ${ }^{-1}$ de materia seca, fue de $15,9 \mathrm{~kg}^{2}$ árbol ${ }^{-1}$ de macronutrimentos $(7,3 \mathrm{~kg}$ de $\mathrm{Ca}, 3,9 \mathrm{~kg}$ de $\mathrm{N}, 2,6 \mathrm{~kg}$ de $\mathrm{K}, 1,0 \mathrm{~kg}$ de $\mathrm{Mg}, 0,7 \mathrm{~kg}$ de $\mathrm{P}$ y 0,4 $\mathrm{kg}$ de S) y de 124 g.árbol $^{-1}$ de micronutrimentos ( $89 \mathrm{~g}$ de Fe, $18 \mathrm{~g}$ de Zn, 9 g de B, 5 g de Mn y 3 $\mathrm{g}$ de $\mathrm{Cu}$ ). El 58\% del $\mathrm{N}$ acumulado se encuentra mayormente en el tronco y las hojas, mientras que el 68\% del $\mathrm{K}$ acumulado se encuentra en tronco, ramas primarias y corteza. La acumulación total de Ca tiene la tendencia a aumentar después del año 7 hasta el 18, mientras que la acumulación total de $\mathrm{K}$ tiene la tendencia a disminuir con la edad a partir del año 7.

\section{LITERATURA CITADA}

ÁVILA R.I. 2003. Evaluación del estado y crecimiento inicial de cuatro especies prioritarias (Pinus maximinoi H.E. Moore, Pinus caribaea, Pinus oocarpa Schiede y Tectona grandis L.f.) del Programa de Incentivos Forestales en la región 2, en los departamentos de alta y Baja Verapaz, Guatemala. Tesis de maestría, CATIE, Turrialba, Costa Rica. 154 p.

BARROSO D., MAZZEI F.A., PEREIRA R.S., CHAHAD V., MENDOÇA L., SILVA L. 2005. Macronutrient deficiency diagnosis in teak seedlings. Árvore 29(5):671-679.

BERTSCH F., CORRALES M., BEJARANO J. 2005. Los Laboratorios de análisis de suelos y foliares en Costa Rica: Informe del Comité de Laboratorios de análisis de suelos, plantas y aguas. Agronomía Costarricense 29(3):125-135.

BLANCO J., IMBERT B., CASTILLO F. 2009. Thinning affects nutrient resorption and nutrient-use efficiency in two Pinus sjlvestris stands in the Pyrenees. Ecological Applications 19(3):682-698.

CHANDRA P.B., SATHRE R., BERGH J., GUSTAVSSON L., LUNDSTRÖ M., HYVÖNEM R. 2011. Potential effects of intensive forestry on biomass production and total carbon balance in north-central Sweden. Environmental Science \& Policy 15:106-124.

DRECHSEL P., ZECH W. 1991. Foliar nutrient levels of broad-leaved tropical trees: a tabular Review. Plant and Soil 131:29-46.

DRECHSEL P., ZECH W. 1994. DRIS evaluation of teak (Tectona grandis L.f.) mineral nutrition and effects of nutrition and site quality on teak growth in West Africa. Forest Ecology and Management 70:121-133.

EGNELL G., AHNLUND K. 2015. Stand productivity following whole-tree harvesting in early thinning of Scots pine stands in Sweden. Forest Ecology and Management 340:40-45.

FAO (ORGANIZACIÓN DE LAS NACIONES UNIDAS PARA LA AGRICULTURA Y LA ALIMENTACIÓN). 1977. Elaboración de una tabla de volumen y un estudio de incremento para Teca (Tectona grandis) en El Salvador. Documento de trabajo $\mathrm{N}^{\circ}$. 14 FO:DP ELS/73/004. 53 p.

GUIMARÃES L., BRITO M., LUIZ DE A.M., BACKES C., AMARAL I. 2010. Influência da adubação potássica no acúmulo de matéria seca em plantas de teca. XXIX Reunião Brasileira de Fertilidade do Solo e Nutrição de Plantas Guarapari - ES, Brasil, 13 a 
17 de setembro de 2010. Centro de Convenções do SESC. 4 p.

HASE H., FOELSTER H. 1983. Impact of plantation forestry with teak (Tectona grandis) on the nutrient status of young aluvial soils in West Venezuela. Forest Ecology and Management 6:33-57.

HENRÍQUEZ C., BERTSCH F., SALAS R. 1995. Fertilidad de suelo: Manual de laboratorio. San José, CR, Asociación Costarricense de la Ciencia del Suelo. $64 \mathrm{p}$.

HERNÁNDEZ R., TORRES A., MÁRQUEZ O., FRANCO W. 1993. Contenido foliar y crecimiento en plantaciones de teca en Ticoporo, Venezuela. Turrialba 43(1):11-15.

KAUL O.N., SHARMA D.C., TANDOM V.N.,SRIVASTAVA P.B.L. 1979. Organic matter and plant nutrients in a teak (Tectona grandis) plantation. Indian Forester 105(8):573-582.

KEOGH R.M. 1979. El futuro de la teca en la América Tropical: Estudio sobre Tectona grandis en el Caribe, Centro América, Venezuela y Colombia. Roma, IT. FAO. Unasylva 31(126):13-19.

KOPPAD A., RAO R. 2003. Effect of moisture conservation methods and fertilizers on nutrient uptake in two-old teak (Tectona grandis L.f.) plantations, pp. 206-211. In: K. Bhat et ál. Proceedings of the International Conference on Quality Timber products of Teak from sustainable forest management. India.

KUMAR J.I.N., KUMAR R.N., KUMAR R., SAJISH P.R. 2009. Quantification of nutrient in the aboveground biomass of teak plantation in a tropical dry deciduous forest of Udaipur, India. Journal of Forest Science 55(6):251-256.

KUMAR P. 2009. Nutrient dynamics of teak plantations and their impact on soil productivity: A case study from India. XIII World Forestry Congress. Buenos Aires, Argentina, 18-23 de Octubre 2009. 11 p.

MARTIARENA R., PINAZO M., VON WALLIS A., PAHR N., KNEBEL O., FERNANDEZ R. 2010. Intensidad de raleo en plantaciones de Pinus taeda L. y sus efectos sobre la conservación de $\mathrm{N}, \mathrm{K}, \mathrm{Ca}$ y $\mathrm{Mg}$. XXII Congreso Argentino de la ciencia del suelo. Rosario, Argentina. 31 de mayo al 4 de junio, 2010. p. 4.

MBAEKWE E., MACKENZIE A. 2008. The use of a best-fit allometric model to estimate aboveground biomass accumulation and distribution in an age series of teak (Tectona grandis L.f.) plantations at Gambari Forest Reserve, Oyo State, Nigeria. Tropical Ecology 49(2):259-270.

MOLLINEDO M. 2003. Relación suelo-planta, factores de sitio y respuesta a la fertilización, en plantaciones jóvenes de teca (Tectona grandis L. f.), en la zona Oeste, Cuenca del Canal de Panamá. Tesis de maestría, CATIE, Costa Rica. 101 p.
MOLLINEDO M., UGALDE L., ALVARADO A., VERJANS J.M., RUDY L. 2005. Relación sueloárbol y factores de sitio, en plantaciones jóvenes de teca (Tectona grandis L.f.), en la Zona oeste de la Cuenca del Canal de Panamá. Agronomía Costarricense 29(1):67-75.

MONTERO M. 1999. Factores de sitio que influyen en el crecimiento de Tectona grandis L.f. y Bombacopsis quinata (Jacq.) Dugand, en Costa Rica. Tesis de maestría, Universidad Austral de Chile, Valdivia, Chile. 77 p.

MONTERO M., KANNINEN M. 2006. Variación de los nutrimentos foliares con la edad de los árboles de Tectona grandis L.f., en Costa Rica. Proyecto: Dinámica de plantaciones. Universidad de Helsinki/ CATIE. Turrialba, Costa Rica. 16 p.

NEGI J. 1990. Biomass production and distribution of nutrients in 20 years old teak (Tectona grandis L.f.) and gamar (Gmelina arborea) plantation in Tripura. The Indian Forester 116(9):681-686.

NEGI J., BAHUGUNA V., SHARMA D. 1995. Biomass and nutrient distribution in young teak (Tectona grandis L.f.) plantations in Tarai Region of Uttar Pradesh. Indian Forester 121(6):455-464.

NWOBOSHI L. 1983. Growth and nutrient requirements in a teak plantation age series in Nigeria: I Linear growth and biomass production. Forest Science 29(1):159-165.

NWOBOSHI L. 1984. Growth and nutrient requirements in a teak plantation age series in Nigeria: II Nutrient accumulation and minimum annual requirements. Forest Science 30(1):35-40.

OSUNDINA M.A., OSONUBI O. 1989. Adventitious roots, leaf abscission and nutrient status of flooded Gmelina and Tectona seedlings. Tree Physiology 5:473-483.

PORTUGUEZ E. 2012. Estimación de la extracción de nutrimentos por parte aérea en plantaciones de teca (Tectona grandis Linn. f.) de las empresas Panamerican Woods y C\&M Investment Group Ltda., en la península de Nicoya, Guanacaste, Costa Rica. Práctica dirigida de licenciatura, Universidad de Costa Rica. San José, Costa Rica. 72 p.

PRASANNA Y., KING P., PRASAD V.S.R.K. 2006. Zinc biosorption on Tectona grandis L.f. leaves biomass: equilibrium and kinetic studies. Chemical Engineering Journal 124:63-70.

SALAS J., INFANTE A. 2006. Producción primaria neta aérea en algunos ecosistemas y estimaciones de biomasa en plantaciones forestales. Rev. For. Lat. 40:47-70

SAUR E., RANGER J., LEMOINE B., GELPE J. 1992. Micronutrient distribution in 16-year-old maritime pine. Tree Physiology 10:307-316.

SEGURA M., CASTILLO A., ALVARADO A., BLANCO F. 2005. Extracción de nutrimentos en plantaciones de 
jaúl (Alnus acuminata) en la Cuenca del río Virilla, Costa Rica. Agronomía Costarricense 29(2):109-120.

STATSOFT INC. 2001. STATISTICA (Data Analysis Software System), versión 6.

THIELE H. 2008. Variables edáficas que afectan el crecimiento de la teca (Tectona grandis L.F.) en la vertiente Pacífica de Costa Rica. Tesis de maestría, Universidad de Costa Rica, San José, Costa Rica. $180 \mathrm{p}$.

TLBG (THE LOUIS BERGER GROUP INC. P). 2006. Informe de la región occidental de la cuenca del canal de Panamá. Presentado a la Autoridad del canal de Panamá. 21 p.

TORRACA S.M., HAAG H.P., MIGLIORINI A.J. 1984. Recrutamento e exportação de nutrimentos por Pinus elliottii var., elliottii em um latossolo vermelho escuro na região de Agudos, SP IPEF 27:41-47.

VAIDES E.E. 2004. Características de sitio que determinan el crecimiento y productividad de teca (Tectona grandis L.f.), en plantaciones forestales de diferentes regiones en Guatemala. Tesis de maestría, CATIE, Turrialba, Costa Rica. 95 p.

VALLEJOS O.S. 1996. Productividad y relaciones del índice de sitio con variables fisiográficas, edafoclimáticas y foliares para Tectona grandis L.f., Bombacopsis quinatum (Jacq) Dugand y Gmelina arborea Roxb. en Costa Rica. Tesis de maestría, CATIE, Turrialba, Costa Rica. 147 p.

VÁSQUEZ W., UGALDE L.A. 1995. Rendimiento y calidad de sitio para Gmelina arborea, Tectona grandis, Bombacopsis quinata y Pinus caribaea, en Guanacaste, Costa Rica. CATIE/ IDA/ FAO/ HOLANDA. Proyecto Madelaña-3. Turrialba, Costa Rica. Serie Técnica, Informe Técnico No.256. 33 p.

VIEIRA E. 2006. Biomass study of Tectona grandis under different spacing conditions in the north of Mato Grosso state. Árvore 30(3):337-341.

VIMAL M., SUDHAKARA K., IAYARAMAN K., SUNANDA C. 2003. Effect of soil-leaf nutritional factors o the productivity of teak (Tectona grandis L.f.) in Kerala State, India, pp. 530-539. In: Proceedings of the International Conference on Quality Timber Products of Teak from Sustainable Forest Management. Peechi, India. 2-5 December. 2003.

ZECH W., KAUPENJOHANN M. 1990. Carences en potassium et phosphore chez Casuarina equisetifolia, Eucalyptus spp. Acacia auriculiformis et Tectona grandis au Sud-Benin (Afrique occidentale). Bois et Forêsts des Tropiques 226:29-36. 